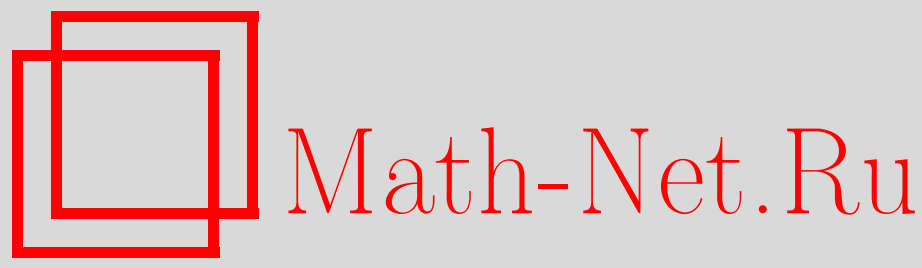

Т. А. Филатова, А. И. Шафаревич, Квазиклассические спектральные серии оператора Шредингера с дельтапотенциалом на прямой и на сфере, ТМФ, 2010, том 164, номер 2, 279-298

DOI: https://doi.org/10.4213/tmf6539

Использование Общероссийского математического портала Math-Net.Ru подразумевает, что вы прочитали и согласны с пользовательским соглашением http://www . mathnet.ru/rus/agreement

Параметры загрузки:

IP : 18.207 .199 .55

26 апреля 2023 г., $17: 30: 45$

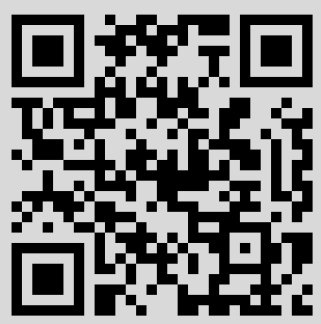




\section{КВАЗИКЛАССИЧЕСКИЕ СПЕКТРАЛЬНЫЕ СЕРИИ ОПЕРАТОРА ШРЕДИНГЕРА С ДЕЛЬТА-ПОТЕНЦИАЛОМ НА ПРЯМОЙ И НА СФЕРЕ}

В квазиклассическом пределе $h \rightarrow 0$ описаны спектральные серии оператора Шредингера $H=-\left(h^{2} / 2\right) \Delta+V(x)+\alpha \delta\left(x-x_{0}\right), \alpha \in \mathbb{R}$, с дельта-потенциалом на действительной прямой, трехмерной и двумерной стандартных сферах. В первом случае рассмотрен гладкий потенциал $V(x)$ такой, что $\lim _{|x| \rightarrow \infty} V(x)=+\infty$. В двух последних случаях $V(x)=0$. Для каждого случая описаны классические траектории, соответствующие в квазиклассическом пределе квантовой задаче с дельта-потенциалом.

Ключевые слова: квазиклассический спектр, оператор Шредингера, дельта-потенциал, лагранжево многообразие, канонический оператор Маслова.

\section{1. ВВЕДЕНИЕ}

Операторы Шредингера с точечными потенциалами (потенциалами нулевого радиуса, дельта-потенциалами) используются во многих физических и математических задачах. Одной из первых физических работ, где для исследования зонного спектра периодических систем применялись потенциалы нулевого радиуса, является известная статья [1]. С помощью метода точечных потенциалов исследовались различные физические явления [2]-[4]. Операторы с дельта-потенциалами также изучались, например, в работах [5]-[8]. Строгое математическое обоснование метода потенциалов нулевого радиуса было дано в статье [9], где было предложено использовать формулу Крейна для описания резольвент операторов с точечными возмущениями. Обширная библиография работ, посвященных применениям метода точечных потенциалов, содержится в монографиях [10], [11]. В работах [12]-[15] на основе теории расширений изучались спектральные свойства опе-

* Институт проблем механики им. А. Ю. Ишлинского РАН, Москва, Россия

${ }^{\dagger}$ Московский физико-технический институт, Москва, Россия.

E-mail: tatyana.filatova@gmail.com

${ }^{\ddagger}$ Московский государственный университет им. М. В. Ломоносова, Москва, Россия. E-mail: shafarev@yahoo.com 
раторов с дельта-потенциалами и близких к ним операторов на сингулярных пространствах.

В настоящей работе обсуждаются квазиклассические свойства простейших операторов с дельта-потенциалами. Квазиклассическая теория для широкого класса уравнений с гладкими коэффициентами была развита в работах Маслова (см., например, монографии [16], [17]); в статье [18] эта теория применялась к спектральной задаче для оператора Лапласа-Бельтрами на $k$-мерной сфере. В работе [19] квазиклассическая теория использовалась для описания асимптотики функции с особенностью (функции Грина) для стационарного уравнения Шредингера.

Мы рассматриваем спектральную задачу

$$
H \psi=E \psi
$$

для оператора Шредингера с дельта-потенциалом,

$$
H=-\frac{h^{2}}{2} \Delta+V(x)+\alpha \delta\left(x-x_{0}\right), \quad \alpha \in \mathbb{R},
$$

в квазиклассическом пределе $h \rightarrow 0$ для трех случаев: $x \in \mathbb{R}, x \in S_{a}^{2}$ и $x \in S_{a}^{3}$, где $S_{a}^{n}$ - стандартная $n$-мерная сфера радиуса $a$. В первом случае рассматривается потенциал $V(x)$ такой, что $\lim _{|x| \rightarrow \infty} V(x)=+\infty$. В двух последних случаях $V(x)=0$. Цель работы - получить квазиклассические спектральные серии оператора (2), а также описать классические траектории и инвариантные лагранжевы многообразия, соответствующие этим сериям.

Структура работы следующая. В разделе 2 приводятся необходимые сведения из теории самосопряженных расширений. В разделе 3 кратко изложены основные сведения о строении спектра оператора с дельта-потенциалом (см. также работы [12], [15], [20]). В разделе 4 с помощью конструкции канонического оператора Маслова [17], а также с помощью изложенных в разделе 3 сведений строятся почти собственные функции оператора $H$, т. е. приближенные с точностью $o(h)$ решения уравнения (1). Почти собственные функции подбираются так, чтобы приближенные решения удовлетворяли заданным граничным условиям (так называемым условиям сшивки, параметризующим данное самосопряженное расширение), которые и будут давать уравнения на квазиклассический спектр задачи(1), (2). Квазиклассические асимптотики спектра оператора (2) описаны в теоремах 1-3 из раздела 4 . Все величины, входящие в полученные асимптотические формулы, выражаются через характеристики некоторого семейства фазовых траекторий; иными словами, в пределе $h \rightarrow 0$ квантовым состояниям отвечают определенные классические движения. Мы описываем классические движения, порожденные дельта-потенциалом; они представляют собой совокупность траекторий гамильтоновой системы со специальными начальными условиями. Семейство этих траекторий образует лагранжево многообразие $\Lambda$. Именно на этих лагранжевых многообразиях строится канонический оператор, а полученные уравнения на квазиклассический спектр являются нестандартными условиями квантования на построенных многообразиях $\Lambda$ (см. раздел 4). 


\section{2. ФОРМАЛЬНОЕ ОПРЕДЕЛЕНИЕ ОПЕРАТОРА $H$}

В теории точечных потенциалов (дельта-потенциалов) нетривиальным является вопрос о придании операторного смысла в пространстве $L_{2}(M)$ (где в нашем случае $M$ есть $\mathbb{R}, S_{a}^{3}$ или $S_{a}^{2}$ ) формальному выражению

$$
H=-\frac{h^{2}}{2} \Delta+V(x)+\alpha \delta\left(x-x_{0}\right), \quad \alpha \in \mathbb{R},
$$

где $\delta(x)$ - дельта-функция Дирака, а $V(x)$ - гладкая функция. Мы пользуемся следующей конструкцией [9]. Рассмотрим самосопряженный оператор

$$
H_{0}=-\frac{h^{2}}{2} \Delta+V(x),
$$

область определения которого есть $D\left(H_{0}\right)=W_{2}^{2}(M)$ (здесь $W_{2}^{2}(M)$ - второе пространство Соболева, при $\operatorname{dim} M \leqslant 3$ справедливо вложение $W_{2}^{2}(M) \subset C(M)$, где $C(M)$ - пространство непрерывных функций на $M)$. На оператор $H$ налагаются следующие естественные условия:

1) оператор $H$ самосопряжен;

2) действие оператора $H$ на функциях $\psi(x)$ таких, что $\psi\left(x_{0}\right)=0$, совпадает с действием оператора $H_{0}$.

Таким образом, $H$ - самосопряженное расширение симметрического оператора $\left.H_{0}\right|_{\psi\left(x_{0}\right)=0}$, получающегося путем ограничения оператора $H_{0}$ на пространство функций, обращающихся в нуль в точке $x_{0}$. Все такие расширения $H$ параметризуются одним параметром $\alpha$ (коэффициентом при дельта-потенциале в (3)) и описываются следующим образом. Область определения оператора $H$ составляют функции $f \in D\left(\left(\left.H_{0}\right|_{\psi\left(x_{0}\right)=0}\right)^{*}\right)$, обладающие следующими свойствами.

1. В окрестности точки $x_{0}$ для них справедливо разложение [12]

$$
f(x)=a(f) F_{0}\left(x, x_{0}\right)+b(f)+R\left(x, x_{0}\right),
$$

где $a(f), b(f) \in \mathbb{C}$

$$
F_{0}\left(x, x_{0}\right)= \begin{cases}-\frac{1}{2} r\left(x, x_{0}\right), & \operatorname{dim} M=1, \\ -\frac{1}{2 \pi} \ln r\left(x, x_{0}\right), & \operatorname{dim} M=2, \\ \frac{1}{4 \pi} \frac{1}{r\left(x, x_{0}\right)}, & \operatorname{dim} M=3 ;\end{cases}
$$

функция $R\left(x, x_{0}\right)$ при $x \rightarrow x_{0}$ имеет асимптотику

$$
R\left(x, x_{0}\right)= \begin{cases}o\left(r\left(x, x_{0}\right)\right), & \operatorname{dim} M=1, \\ o(1), & \operatorname{dim} M=2, \quad \operatorname{dim} M=3 .\end{cases}
$$

Здесь и далее $r\left(x, x_{0}\right)$ - расстояние между точками $x$ и $x_{0}$ на $M$. 
2. Для таких функций $f(x)$ (для данного оператора $H$ ) должно выполняется граничное условие

$$
a(f)=\frac{2 \alpha}{h^{2}} b(f)
$$

ПримеР. Найдем область определения самосопряженного расширения $H$ оператора $H_{0}$ в случае $x \in \mathbb{R}$. Согласно (5)-(7) функция $f \in D(H)$ имеет вид

$$
f(x)=a(f)\left(-\frac{\left|x-x_{0}\right|}{2}\right)+b(f)+o\left(\left|x-x_{0}\right|\right) .
$$

В одномерном случае коэффициенты $a(f), b(f)$ легко вычисляются:

$$
a(f)=f^{\prime}\left(x_{0}-0\right)-f^{\prime}\left(x_{0}+0\right), \quad b(f)=f(0) .
$$

Поэтому в силу (8) получаем граничное условие [12]

$$
f^{\prime}\left(x_{0}-0\right)-f^{\prime}\left(x_{0}+0\right)=\frac{2 \alpha}{h^{2}} f(0),
$$

причем в точке $x_{0}$ функция $f(x)$ непрерывна,

$$
f\left(x_{0}-0\right)=f\left(x_{0}+0\right) .
$$

Следовательно, область определения самосопряженного расширения $H$ оператора $\left.H_{0}\right|_{\psi(0)=0}$ состоит из функций $f \in D\left(\left(\left.H_{0}\right|_{\psi\left(x_{0}\right)=0}\right)^{*}\right)$, для которых выполняются условия (10), (11), причем при $x<x_{0}$ и при $x>x_{0}$ производная $f^{\prime}(x)$ абсолютно непрерывна.

\section{3. СПЕКТР ТОЧЕЧНОГО ВОЗМУЩЕНИЯ}

Пусть $M=S_{a}^{2}$ или $M=S_{a}^{3}, V(x)=0$. Спектр точечного возмущения лапласиана выражается в терминах Q-функции Крейна [12], [15]. Пусть $G^{0}(x, y ; z)$ - функция Грина введенного в разделе 2 оператора $-\Delta$ на $M$, т. е. интегральное ядро оператора $(-\Delta-z)^{-1}$ (здесь $z \in \rho(-\Delta)$, где $\rho(-\Delta)-$ резольвентное множество оператора $\left.-\Delta\right)$ :

$$
(-\Delta-z)^{-1} f(x)=\int_{M} G^{0}(x, y ; z) f(y) d \omega,
$$

где $d \omega$ - форма объема на $M$. Пусть $G_{\mathrm{reg}}^{0}(x, y ; z)=G^{0}(x, y ; z)+F_{0}(x, y)$ - регуляризованная функция Грина, функция $F_{0}(x, y)$ определена в формуле $(6)$. По определению Q-функция Крейна есть $Q\left(z ; x_{0}\right)=G_{\mathrm{reg}}^{0}\left(x_{0}, x_{0} ; z\right)$.

Далее мы опишем спектр оператора $H$ при фиксированном $x_{0}$, опираясь на результаты работы [15]. В рассматриваемых задачах спектр $\operatorname{spec}\left(H_{0}\right)$ оператора $H(0)$, приведенного в (4), дискретный: состоит из неограниченной последовательности $\lambda_{0}<\lambda_{1}<\cdots<\lambda_{n}<\cdots$ собственных значений с конечными кратностями $k_{0}, k_{1}, \ldots$ $\ldots, k_{n}, \ldots$. Каждое собственное значение $\lambda_{n}$ оператора $H_{0}$ кратности $k_{n}$ является собственным значением оператора $H$ кратности $k_{n}-1, k_{n}$ или $k_{n}+1$ (если $k_{n}=1$, то кратность, равная $k_{n}-1$, означает, что $\lambda_{n}$ не лежит в $\left.\operatorname{spec} H\right)$. Значение $\lambda \notin \operatorname{spec} H_{0}$ 
является собственным значением оператора $H$ тогда и только тогда, когда $z=2 \lambda / h^{2}$ есть решение уравнения

$$
Q\left(z ; x_{0}\right)-\frac{h^{2}}{2 \alpha}=0
$$

Данная формула является универсальной для описания вклада в спектр, вносимого дельта-потенциалом. Таким образом (см. теорему 2.5 в работе [15]), спектр оператора $H$ будет состоять из кратных $\left(k_{n}>1\right)$ собственных значений оператора $H_{0}$, а также из значений $\lambda=\varepsilon_{n}$, отвечающих решениям $z=2 \lambda / h^{2}$ уравнения (12). В указанной работе [15] также сформулирован результат для собственных функций оператора $H$. Собственная функция $\psi$ оператора $H_{0}$ является собственной функцией оператора $H$, если $\psi\left(x_{0}\right)=0$ (в частности, такие функции всегда существуют, если собственное значение оператора $H_{0}$ кратное). Помимо собственных функций оператора $H_{0}$, у оператора $H$ есть еще следующие собственные функции. Обозначим $G^{0}\left(z, x_{0} ; \varepsilon_{n}\right)$ через $g_{x_{0}}(z)$, тогда эти дополнительные (нормированные) собственные функции вычисляются по формуле

$$
\Phi_{n}=\left[\frac{\partial Q}{\partial z}\left(\varepsilon_{n} ; x_{0}\right)\right]^{-1} g_{x_{0}}\left(\varepsilon_{n}\right)
$$

\section{4. КВАЗИКЛАССИЧЕСКИЕ СПЕКТРАЛЬНЫЕ СЕРИИ ОПЕРАТОРА $H$}

В этом разделе мы найдем спектр задачи

$$
\left(-\frac{h^{2}}{2} \Delta+V(x)+\alpha \delta\left(x-x_{0}\right)\right) \psi=E \psi, \quad \alpha \in \mathbb{R}
$$

в квазиклассическом пределе $h \rightarrow 0$ на действительной прямой, трехмерной и двумерной сферах и опишем соответствующие инвариантные лагранжевы многообразия.

4.1. Одномерный случай. Определим, какие классические движения соответствуют задаче

$$
\left(-\frac{h^{2}}{2} \frac{d^{2}}{d x^{2}}+V(x)+\alpha \delta\left(x-x_{0}\right)\right) \psi=E \psi
$$

при $h \rightarrow 0$, где $\lim _{|x| \rightarrow \infty} V(x)=+\infty, x \in \mathbb{R}, \alpha \in \mathbb{R}_{+}$. Рассмотрим классические движения, соответствующие квантовой задаче с $\alpha=0$ (см. рис. 1):

$$
\left(-\frac{h^{2}}{2} \frac{d^{2}}{d x^{2}}+V(x)\right) \psi=E \psi
$$




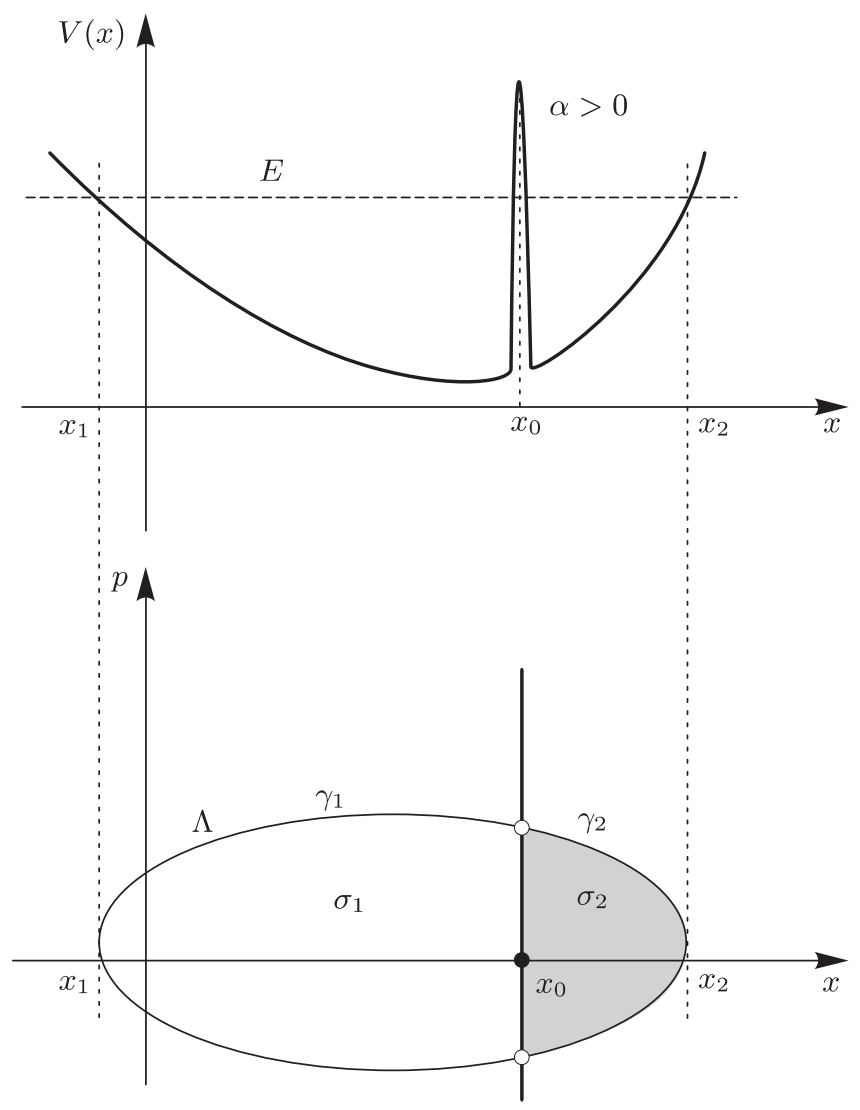

Рис. 1. Классические движения в случае квантовой задачи (14), где $V\left(x_{1}\right)=V\left(x_{2}\right)=E, x_{1}<x_{0}<x_{2}$.

Для данной задачи мы имеем замкнутую классическую траекторию $p^{2}+V(x)=E$. Для задачи (14) при $\alpha \neq 0$ отличие состоит в том, что классическая траектория, соответствующая задаче (15), делится вертикальной прямой, проходящей через точку $x_{0}$, на две кривые $\gamma_{1}$ и $\gamma_{2}$ (рис. 1); через интегралы вдоль этих кривых и через площади $\sigma_{1}$ и $\sigma_{2}$ областей, ограниченных этими кривыми, выражаются в данном случае уравнения на спектр задачи (14). Именно совокупность кривых $\gamma_{1}$ и $\gamma_{2}$ является в данном случае инвариантным лагранжевым многообразием $\Lambda$, описывающим классические движения.

Справедлива следующая теорема.

Tеорема 1. Пусть существует число $E=O(1)$, удовлетворяющее с точностью $O(h)$ следующим уравнениям, где $p(x)=\sqrt{2(E-V(x))}:$ при $\alpha=O(1)-$ одному из уравнений

$$
\int_{x_{0}}^{x_{2}} p(x) d x=\pi h\left(n+\frac{1}{4}\right), \quad \int_{x_{1}}^{x_{0}} p(x) d x=\pi h\left(k+\frac{3}{4}\right) ;
$$


при $\alpha=\alpha_{0} h=O(h)-$ уравнению

$$
\begin{aligned}
& \sin \left(\frac{1}{2 h} \oint_{\Gamma: p^{2} / 2+V(x)=E} p(x) d x+\frac{\pi}{2}\right)= \\
& \quad=-\frac{\alpha_{0}}{p\left(x_{0}\right)} \cos \left(\frac{1}{2 h} \oint_{\Gamma: p^{2} / 2+V(x)=E} p(x) d x+\frac{\pi}{2}\right)-\cos \left(\frac{\sigma_{2}-\sigma_{1}}{2 h}\right),
\end{aligned}
$$

где $x_{1}<x_{0}<x_{2}$,

$$
\sigma_{1}=2 \int_{x_{0}}^{x_{1}} \sqrt{2(E-V(x))} d x, \quad \sigma_{2}=2 \int_{x_{0}}^{x_{2}} \sqrt{2(E-V(x))} d x
$$

при $\alpha=O\left(h^{1+\delta}\right), \delta>0,-$ уравнению

$$
\oint_{\Gamma: p^{2} / 2+V(x)=E} p(x) d x=2 \pi h\left(n+\frac{1}{2}\right),
$$

где $n, k \in \mathbb{Z}_{+}(n, k \sim 1 / h, h \rightarrow 0)$.

Тогда существует собственное значение $E_{0}$ оператора $H$ такое, что $\left|E-E_{0}\right|=$ $O\left(h^{2}\right)$ nрu $h \rightarrow 0$.

Смысл приведенных формул легко понять. Действительно, если дельта-возмущение велико, т. е. $\alpha=O(1)$ при $h \rightarrow 0$, то уравнение, определяющее спектр, разбивается на два независимых условия квантования, соответствующих движению в правой и левой частях потенциальной ямы. В случае слабого дельта-возмущения, а именно при $\alpha=o(h)$, наличие такого возмущения не вносит ощутимого вклада в спектр; при этом получается стандартное условие квантования Бора-Зоммерфельда (см., например, книгу [21]), которое справедливо, если дельта-возмущение отсутствует. Случай $\alpha=O(h)$ промежуточный: движения слева и справа от дельта-потенциала взаимодействуют; условие квантования отличается от стандартного правила Бора-Зоммерфельда.

ЗАмЕчАниЕ 1. Соответствующие формулы для симметричной ямы (при $\left.\sigma_{1}=\sigma_{2}\right)$ известны.

ДокАЗАТЕЛЬСтво. Построим функцию, которая, во-первых, при $x>x_{0}$ и при $x<x_{0}$ удовлетворяет уравнению (15) с точностью $O\left(h^{2}\right)$ и, во-вторых, лежит в области определения оператора $H$ задачи (14). Чтобы найти такую функцию, убывающую при $|x| \rightarrow \infty$, воспользуемся конструкцией канонического оператора Маслова [17]. Разобьем область изменения переменной $x$ на два промежутка $L_{1}=\left(-\infty, x_{0}\right)$ и $L_{2}=\left(x_{0},+\infty\right)$. Построим с точностью $O\left(h^{2}\right)$ решения $\psi_{1}(x)$ и $\psi_{2}(x)$ задачи $(15)$ в областях $L_{1}$ и $L_{2}$, убывающие при $|x| \rightarrow \infty$. Искомую функцию запишем в виде

$$
\psi(x)= \begin{cases}\psi_{1}(x), & x \in L_{1} \\ \psi_{2}(x), & x \in L_{2}\end{cases}
$$

Пусть кривая $\Lambda$ (одномерное лагранжево многообразие) определяется уравнением $H_{0}(p, x)=E$, где $H_{0}=p^{2} / 2+V(x)$. Введем на $\Lambda$ параметр $t \in \mathbb{R}$ и форму объема $d t$. 
Рассмотрим область $L_{1}$. Этой области соответствует часть замкнутой лагранжевой кривой $\Lambda$, обозначенная через $\gamma_{1}$ (см. рис. 1). Построим в этой области решение $\psi_{1}(x)$ с помощью конструкции канонического оператора Маслова [17]: $\psi_{1}(x)=$ $K_{1 \Lambda}[1]$, где $K_{1 \Lambda}[1]$ - канонический оператор Маслова на кривой $\gamma_{1}$, примененный к 1. Для этого покроем кривую $\gamma_{1}$ картами $V_{1}, V_{2}, V_{3}$ и введем разбиение единицы по данным картам: $e_{j}(t) \in C^{\infty}(\mathbb{R}), j=1,2,3, \sum_{j=1}^{3} e_{j}(t)=1$. Это решение в неособых картах $V_{1}, V_{3}$ определяется следующей формулой:

$$
\begin{aligned}
\psi_{1}(x)= & K_{1 \Lambda}[1]=J(x)\left\{\exp \left(\frac{i}{h} \int_{x_{0}}^{x} \sqrt{2(E-V(q))} d q\right) e_{1}+\right. \\
& \left.+i \exp \left(\frac{2 i}{h} \int_{x_{1}}^{x_{0}} \sqrt{2(E-V(q))} d q\right) \exp \left(-\frac{i}{h} \int_{x_{0}}^{x} \sqrt{2(E-V(q))} d q\right) e_{3}\right\},
\end{aligned}
$$

где $J(x)=d p / d x=\left(2(E-V(x))^{-1 / 4}\right.$. Для канонического оператора Маслова справедливо коммутационное соотношение [17]

$$
H_{0} K_{\Lambda}[\phi]=K_{\Lambda}\left(\left.H_{0}\right|_{\Lambda} \phi-i h \dot{\phi}\right)+O\left(h^{2}\right)
$$

где $\phi \in C_{0}^{\infty}(\Lambda)$ - произвольная бесконечно дифференцируемая компактная функция на лагранжевом многообразии $\Lambda, \dot{\phi}$ - производная этой функции вдоль траектории. Для $\phi=1$ это соотношение принимает следующий вид:

$$
H_{0} K_{\Lambda}[1]=K_{\Lambda}\left(\left.H_{0}\right|_{\Lambda}\right)+O\left(h^{2}\right) .
$$

Рассмотрим теперь область $L_{2}$, которой соответствует часть замкнутой кривой $\Lambda$, обозначенная через $\gamma_{2}$ (см. рис. 1 ). Аналогичным образом построим решение $\psi_{2}(x)=$ $K_{2 \Lambda}[1]$, где $K_{2 \Lambda}[1]$ - канонический оператор Маслова на кривой $\gamma_{2}$, примененный к $\varphi=1$. Для этого покроем кривую $\gamma_{2}$ картами $V_{4}, V_{5}, V_{6}$ и введем разбиение единицы по данным картам: $e_{j}(t) \in C^{\infty}, j=4,5,6, \sum_{j=4}^{6} e_{j}(t)=1$. Тогда искомое решение в неособых картах $V_{4}, V_{6}$ определяется формулой

$$
\begin{aligned}
\psi_{2}(x)= & K_{2 \Lambda}[1]=J(x)\left\{\exp \left(-\frac{i}{h} \int_{x_{0}}^{x} \sqrt{2(E-V(q))} d q\right) e_{4}+\right. \\
& \left.+i \exp \left(\frac{2 i}{h} \int_{x_{0}}^{x_{2}} \sqrt{2(E-V(q))} d q\right) \exp \left(\frac{i}{h} \int_{x_{0}}^{x} \sqrt{2(E-V(q))} d q\right) e_{6}\right\}
\end{aligned}
$$

и также удовлетворяет условию коммутации (22).

Приведем выражения (21), (23) к следующему виду:

$$
\begin{aligned}
\psi_{1}(x)= & C_{1} \cdot J(x) \exp \left(\frac{i}{h} \int_{x_{1}}^{x_{0}} \sqrt{2(E-V(q))} d q+\frac{i \pi}{4}\right) \times \\
& \times \cos \left\{\frac{1}{h} \int_{x_{0}}^{x} \sqrt{2(E-V(q))} d q-\left(\frac{1}{h} \int_{x_{1}}^{x_{0}} \sqrt{2(E-V(q))} d q+\frac{\pi}{4}\right)\right\}, \\
\psi_{2}(x)= & C_{2} \cdot J(x) \exp \left(\frac{i}{h} \int_{x_{0}}^{x_{2}} \sqrt{2(E-V(q))} d q+\frac{i \pi}{4}\right) \times \\
& \times \cos \left\{\frac{1}{h} \int_{x_{0}}^{x} \sqrt{2(E-V(q))} d q+\left(\frac{1}{h} \int_{x_{0}}^{x_{2}} \sqrt{2(E-V(q))} d q+\frac{\pi}{4}\right)\right\},
\end{aligned}
$$


где $C_{1}, C_{2}=$ const. Итак, в соответствии с (20) построена функция $\psi(x)$, при $x>x_{0}$ и $x<x_{0}$ удовлетворяющая уравнению $H_{0} \psi=E \psi+O\left(h^{2}\right)$ в силу $(22)$.

Далее, для того чтобы $\psi(x)$ принадлежала области определения $D(H)$, необходимо так подобрать коэффициенты $C_{1}, C_{2}$ в $(24)$, чтобы функции $\psi_{1}(x)$ и $\psi_{2}(x)$ удовлетворяли условиям сшивки в точке $x_{0}$ (и тем самым функция $\psi(x)$ принадлежала области определения оператора $H)$ :

$$
\psi_{1}\left(x_{0}-0\right)=\psi_{2}\left(x_{0}+0\right), \quad \psi_{2}^{\prime}\left(x_{0}+0\right)-\psi_{1}^{\prime}\left(x_{0}-0\right)=\frac{2 \alpha}{h^{2}} \psi_{2}\left(x_{0}\right) .
$$

Положим $C_{1}=C, C_{2}=1$ и введем следующие обозначения:

$$
\xi_{1}=\frac{\sigma_{1}}{2 h}+\frac{\pi}{4}, \quad \xi_{2}=\frac{\sigma_{2}}{2 h}+\frac{\pi}{4},
$$

где $\sigma_{1}$ и $\sigma_{2}$ заданы в формуле (18), и

$$
I(x)=\frac{1}{h} \int_{x_{0}}^{x} \sqrt{2(E-V(q))} d q .
$$

Подставим полученные в (24) решения $\psi_{1}(x), \psi_{2}(x)$ в систему (25). Выразив из первого уравнения системы константу $C$ и подставив ее во второе уравнение, получим

$$
\begin{aligned}
2 J^{\prime}\left(x_{0}\right) e^{i \xi_{2}} \cos \left(I\left(x_{0}\right)+\xi_{2}\right)-2 J\left(x_{0}\right) I^{\prime}\left(x_{0}\right) e^{i \xi_{2}} \sin \left(I\left(x_{0}\right)+\xi_{2}\right)- \\
\quad-2 C J^{\prime}\left(x_{0}\right) e^{i \xi_{1}} \cos \left(I\left(x_{0}\right)-\xi_{1}\right)+2 J\left(x_{0}\right) I^{\prime}\left(x_{0}\right) e^{i \xi_{1}} \sin \left(I\left(x_{0}\right)-\xi_{1}\right)= \\
=\frac{2 \alpha}{h^{2}} J\left(x_{0}\right) e^{i \xi_{2}} \cos \left(I\left(x_{0}\right)+\xi_{2}\right) .
\end{aligned}
$$

Рассматривая отдельно случаи $\alpha=O(1), \alpha=O(h)$ и $\alpha=O\left(h^{1+\delta}\right), \delta>0$, получаем уравнения на спектр, описанные в теореме.

Итак, мы построили удовлетворяющие условиям сшивки (25) функции $\psi_{1}(x)$, $\psi_{2}(x)$, содержащие константы $C_{1}, C_{2}$, и тем самым функция (20) обладает следующими свойствами: $\psi(x) \in D(H)$ и $H \psi=E_{0} \psi+O\left(h^{2}\right)$, в силу выполнения коммутационного соотношения (22) для функций $\psi_{1}, \psi_{2}$, где $E_{0}=\left.H_{0}\right|_{\Lambda}$. Для завершения доказательства воспользуемся хорошо известным утверждением (см., например, книгу [17]).

ЛЕмма. Пусть

$$
\left(H-E_{0}\right) \psi=f, \quad(H-E) \psi=0, \quad \psi \in D(H) .
$$

Тогда $\left|E-E_{0}\right| \leqslant\|f\|$.

Итак, из равенства $\left(H-E_{0}\right) \psi=O\left(h^{2}\right)$ следует, что $\left(E-E_{0}\right) \psi=O\left(h^{2}\right)$.

4.2. Трехмерный случай. Рассмотрим при $h \rightarrow 0$ спектральную задачу на трехмерной сфере $S_{a}^{3}$ радиуса $a$ :

$$
\left(-\frac{h^{2}}{2} \Delta+\alpha \delta\left(x-x_{0}\right)\right) \psi=E \psi, \quad x \in S_{a}^{3} .
$$


Определим объект, в терминах которого выражаются квазиклассические спектральные серии - лагранжево многообразие $\Lambda$, описывающее искомые классические движения, соответствующие квантовой задаче (27).

Пусть $(x, p) \in T^{*} S_{a}^{3}$, где $T^{*} S_{a}^{3}$ - кокасательное расслоение к сфере. Рассмотрим траектории (см. рис. 2)

$$
x=X(\omega, t), \quad p=P(\omega, t), \quad \omega \in S_{\sqrt{2 E}}^{2}, \quad t \in \mathbb{R},
$$

где $S_{\sqrt{2 E}}^{2}-$ двумерная сфера радиуса $\sqrt{2 E}$, гамильтоновой системы

$$
\dot{x}=\frac{\partial H}{\partial p}, \quad \dot{p}=-\frac{\partial H}{\partial x}, \quad H=\frac{p^{2}}{2},
$$

с начальными условиями

$$
x(0)=x_{0}, \quad p(0)=\omega, \quad|\omega|=\sqrt{2 E} .
$$

Другими словами, из точки $x_{0}$ (в которой находится дельта-потенциал) по поверхности сферы $S_{a}^{3}$ с импульсом $p \in \Lambda_{0}$, где $\Lambda_{0}=\left\{x=x_{0},|p|=\sqrt{2 E}\right\}$, выпускаются траектории гамильтоновой системы; в результате получается многообразие $\Lambda=\bigcup_{t} g_{t} \Lambda_{0}$, диффеоморфное $S^{2} \times S^{1}$. Это многообразие и описывает нужные классические движения. Проекции траекторий на сферу $S_{a}^{3}$ суть геодезические (большие круги). Многообразие $\Lambda$ имеет единственный цикл $\Gamma$; в качестве этого цикла можно взять замкнутую траекторию гамильтоновой системы (29) с начальными условиями (30) (см. рис. 2).

Проекция многообразия $\Lambda$ на $x$-пространство устроена следующим образом. В каждую точку сферы $S_{a}^{3}$ за исключением точки $x_{0}$ и точки $x^{\prime}$, диаметрально противоположной $x_{0}$, проецируются две точки многообразия $\Lambda$, а именно $(x, p)$ и $(x,-p)$ (это соответствует тому, что из фиксированной точки $x_{0}$ в любую другую точку $x \in S_{a}^{3}$, $x \neq x^{\prime}$, можно попасть, двигаясь по сфере, двумя путями: по малой и по большой дугам большого круга). При этом в точки $x_{0}$ и $x^{\prime}$ сферы $S_{a}^{3}$ проецируется вся двумерная сфера $S_{\sqrt{2 E}}^{2}$. Условие квантования на многообразии $\Lambda$ вдоль цикла Г и есть искомое уравнение, определяющее спектр задачи (27).

Если из многообразия $\Lambda$ выкинуть сферу $S_{\sqrt{2 E}}^{2}$ над точкой $x_{0}$, то получится некомпактное многообразие $\Lambda^{\prime}=\Lambda \backslash S^{2}$. Многообразие $\Lambda^{\prime}$ определяет собственную функцию.

Справедлива следующая теорема.

ТЕОрема 2. Пусть число $E=O(1)$ удовлетворяет с точностъю $O(h)$ следующим уравнениям: при $h^{3} / \alpha \rightarrow 0$ - уравнению

$$
\oint_{\Gamma}(p, d x)=2 \pi h\left(k+\frac{1}{2}\right), \quad k \in \mathbb{Z}_{+} \quad\left(k \sim \frac{1}{h}, h \rightarrow 0\right)
$$




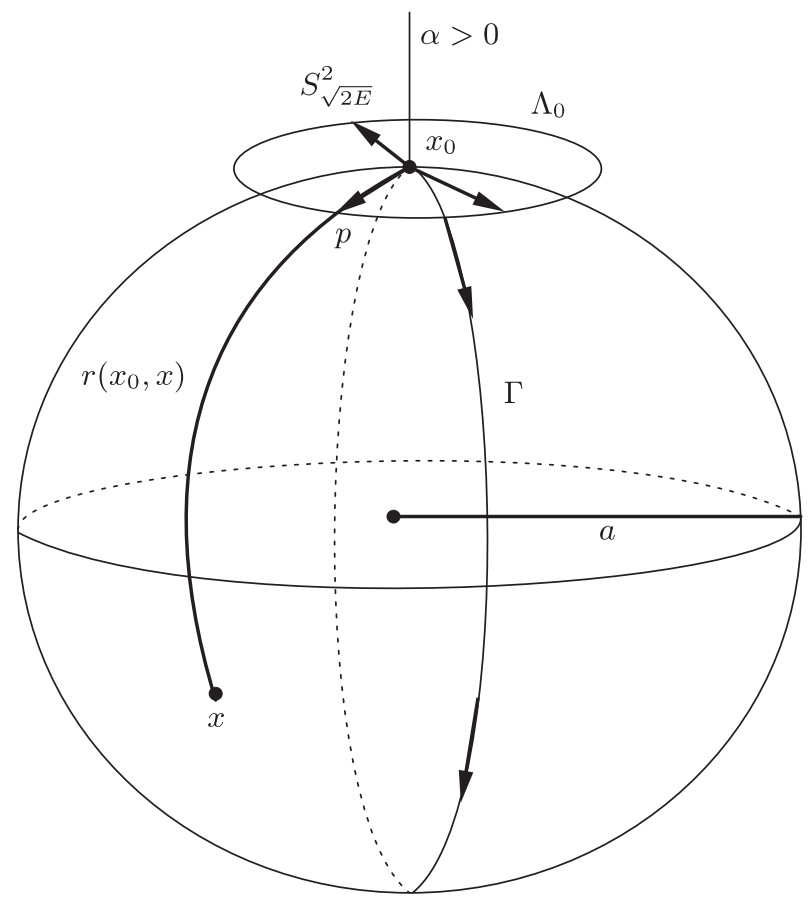

Рис. 2. Классические траектории на сфере $S_{a}^{3}$ (геодезические), соответствующие квантовой задаче (27) (схематично).

при $h^{3} / \alpha=O(1)\left(\right.$ m.е. при $\left.\alpha=\alpha_{0} h^{3}\right)$ - уравнению

$$
\operatorname{ctg}\left(\frac{1}{2 h} \oint_{\Gamma}(p, d x)\right)=-\frac{2 \pi}{\alpha_{0} \sqrt{2 E}}
$$

при $h^{3} / \alpha \rightarrow \infty-$ уравнению

$$
\oint_{\Gamma}(p, d x)=2 \pi h k
$$

Тогда существуют собственное значение $E_{0}$ оператора $H$ maкое, что $\left|E-E_{0}\right|=$ $O\left(h^{2}\right)$, и функиия $\psi$ такая, что $H \psi=E \psi+O\left(h^{2}\right)$. При этом функиия $\psi$ выражается через канонический оператор Маслова на многообразии $\Lambda^{\prime}: \psi=K_{\Lambda^{\prime}}[1]$. Вне точки $x_{0}$ и диаметрально противоположной ей точки $x^{\prime}$ собственная фуункия определяется выражением

$$
\psi=\frac{C}{a \sin \frac{r\left(x, x_{0}\right)}{a}} \sin \left\{\frac{1}{2 h}\left(\int_{x_{0}}^{x}(p, d x)-2 \pi a \sqrt{2 E}\right)\right\}+O(h),
$$

где $C=$ const.

5 Теоретическая и математическая физика, т. 164, № 2, 2010 г. 
ЗАмечАниЕ 2. Интегралы в теореме 2 можно вычислить: имеем, соответственно, для уравнений (31)-(33)

$$
\begin{aligned}
& E=\frac{h^{2}}{2 a^{2}}\left(k+\frac{1}{2}\right)^{2} \quad \text { при } \quad \frac{h^{3}}{\alpha} \rightarrow 0 ; \\
& \operatorname{ctg}\left(\frac{\pi a}{h} \sqrt{2 E}\right)=-\frac{2 \pi}{\alpha_{0} \sqrt{2 E}} \quad \text { при } \quad \frac{h^{3}}{\alpha}=O(1) \quad\left(\alpha=\alpha_{0} h^{3}\right) \text {; } \\
& E=\frac{h^{2}}{2 a^{2}} k^{2} \quad \text { при } \quad \frac{h^{3}}{\alpha} \rightarrow \infty .
\end{aligned}
$$

ЗАмЕчаниЕ 3 . Собственная функция $\psi$ имеет особенность в точке $x_{0}$, что является следствием некомпактности многообразия $\Lambda^{\prime}$.

ЗАмечание 4. Описанное в теореме уравнение (31) совпадает со стандартным условием Бора-Зоммерфельда-Маслова на многообразии $\Lambda$, которое имеет вид (см., например, книгу [17])

$$
\frac{1}{2 \pi h} \oint_{\gamma}(p, d x)=m+\frac{\operatorname{Ind} \gamma}{4}
$$

где $\gamma$ - цикл на $\Lambda$, Ind $\gamma$ - индекс Маслова на $\Lambda, m \in \mathbb{Z}_{+}(m \sim 1 / h$ при $h \rightarrow 0)$.

ДокАзАтЕльство. Точное уравнение на спектр в случае трехмерной сферы имеет вид (12), где

$$
Q\left(E ; x_{0}\right)=-\frac{\sqrt{2 a^{2} E / h^{2}+1}}{4 \pi a} \operatorname{ctg}\left(\pi \sqrt{\frac{2 a^{2} E}{h^{2}}+1}\right)
$$

- Q-функция Крейна в случае трехмерной сферы [12].

Рассмотрим асимптотику уравнения (12) при $h \rightarrow 0$. Учитывая, что

$$
\left.\left(\sqrt{\frac{2 a^{2} E}{h^{2}}+1}\right)\right|_{h \rightarrow 0}=\left.\left(\frac{a \sqrt{2 E}}{h} \sqrt{1+\frac{h^{2}}{2 a^{2} E}}\right)\right|_{h \rightarrow 0}=\frac{a \sqrt{2 E}}{h}+O(h),
$$

получаем с точностью $O(h)$ условие квантования на лагранжевом многообразии $\Lambda$ для случая трехмерной сферы:

$$
-\frac{\sqrt{2 E}}{4 \pi h} \operatorname{ctg}\left(\frac{\pi a \sqrt{2 E}}{h}\right)-\frac{h^{2}}{2 \alpha}=0
$$

или, в привычном виде,

$$
\operatorname{ctg}\left(\frac{\pi a \sqrt{2 E}}{h}\right)=-\frac{2 \pi h^{3}}{\alpha \sqrt{2 E}} .
$$

Условия квантования, изложенные в теореме 2 , получены из данного уравнения при различных соотношениях между $\alpha$ и $h^{3}$.

На трехмерной сфере точное выражение для собственной функции, соответствующей решениям $z=E$ уравнения (12), согласно формуле (13) имеет вид [12]

$$
\begin{aligned}
\psi\left(x ; x_{0}, h, E\right)= & \frac{1}{4 \pi a \sin \frac{r\left(x, x_{0}\right)}{a}}\left\{\cos \left(\frac{r\left(x, x_{0}\right)}{a} I(h, E)\right)-\right. \\
& \left.-\sin \left(\frac{r\left(x, x_{0}\right)}{a} I(h, E)\right) \operatorname{ctg}(\pi I(h, E))\right\},
\end{aligned}
$$


где

$$
I(h, E)=\frac{a \sqrt{2 E}}{h} \sqrt{1+\frac{h^{2}}{2 a^{2} E}}
$$

Теперь получим асимптотику с точностью $O(h)$ собственной функции $(35)$ при $h \rightarrow 0$. Принимая во внимание разложение $I(h, E)=a \sqrt{2 E} / h+O(h)$, приходим к формуле

$$
\begin{aligned}
\psi\left(x ; x_{0}, h, E\right)= & \frac{1}{4 \pi a \sin \frac{r\left(x, x_{0}\right)}{a}}\left\{\cos \left(\frac{r\left(x, x_{0}\right) \sqrt{2 E}}{h}\right)-\right. \\
& \left.-\sin \left(\frac{r\left(x, x_{0}\right) \sqrt{2 E}}{h}\right) \operatorname{ctg}\left(\frac{\pi a \sqrt{2 E}}{h}\right)\right\} .
\end{aligned}
$$

Данное выражение для собственной функции при подстановке в него условия (34) приводится к следующему виду:

$$
\begin{aligned}
\psi\left(x ; x_{0}, h, E\right)= & \frac{1}{4 \pi a \sin \frac{r\left(x, x_{0}\right)}{a}} \times \\
& \times \cos \left\{\frac{r\left(x, x_{0}\right) \sqrt{2 E}}{h}-\operatorname{arctg}\left(\frac{2 \pi h^{3}}{\alpha \sqrt{2 E}}\right)\right\}+O(h) .
\end{aligned}
$$

При выполнении условия квантования (34) функция (36) выражается через канонический оператор Маслова: $\psi=K_{\Lambda^{\prime}}[1]$ на многообразии $\Lambda^{\prime}$. Покажем это.

Методом канонического оператора Маслова [17] построим решение уравнения

$$
-\frac{h^{2}}{2} \Delta \psi=E \psi+O\left(h^{2}\right)
$$

в неособых картах, а именно вне окрестностей точки $x_{0}$ и диаметрально противоположной ей точки $x^{\prime}$. Неособых карт на многообразии $\Lambda^{\prime}$ всего две. Они выбираются так, чтобы точки многообразия $\Lambda^{\prime}$ из первой и второй карт имели противоположные импульсы. Это соответствует тому, что из точки $x_{0} \in S_{a}^{3}$ в любую точку $x$ можно попасть по двум траекториям на сфере $S_{a}^{3}$, по малой и по большой дугам большого круга, выпуская из точки $x_{0}$ векторы импульсов противоположных направлений $p$ и $-p$. В итоге получаем следующее выражение для канонического оператора:

$$
\begin{aligned}
K_{\Lambda^{\prime}}[1]= & \frac{1}{\sqrt{J}}\left\{\exp \left(i \frac{r\left(x, x_{0}\right) \sqrt{2 E}}{h}\right)+\right. \\
& \left.+\exp \left(-i \frac{r\left(x, x_{0}\right) \sqrt{2 E}}{h}\right) \exp \left(\frac{2 i}{h}\left(\pi a \sqrt{2 E}+\frac{\pi h}{2}\right)\right)\right\}
\end{aligned}
$$

где

$$
r\left(x, x_{0}\right) \sqrt{2 E}=\int_{x_{0}}^{x}(p, d x)
$$


есть результат вычисления интеграла вдоль траектории $\Gamma$, а $J=a^{2} \sin ^{2} \frac{r\left(x, x_{0}\right)}{a}-$ якобиан. После несложных преобразований выражения (39) приходим к формуле

$$
\begin{aligned}
K_{\Lambda^{\prime}}[1]= & \frac{2}{\left|a \sin \frac{r\left(x, x_{0}\right)}{a}\right|} \exp \left(i \frac{\pi a \sqrt{2 E}}{h}\right) \times \\
& \times \cos \left(\frac{r\left(x, x_{0}\right) \sqrt{2 E}}{h}-\frac{\pi a \sqrt{2 E}}{h}-\frac{\pi}{2}\right) .
\end{aligned}
$$

Как видно, данное решение, построенное методом канонического оператора Маслова, при $x \rightarrow x_{0}$ имеет тот же вид и ту же особенность в якобиане, что и функция (36), следовательно, функция (40) принадлежит области определения оператора $H$.

Приравнивая аргументы косинуса в выражениях (37) и (40), приходим в точности к условию квантования (34). Следовательно, вне точки $x_{0}$ и диаметрально противоположной ей точки $x^{\prime}$ при выполнении приведенных в теореме условий квантования с точностью $O(h)$ выполняется равенство $\psi=K_{\Lambda^{\prime}}[1]$.

Для функции $\psi(x)$ такой, что $\psi(x)=K_{\Lambda^{\prime}}[1]$, выполняется коммутационное соотношение (21), откуда следует справедливость уравнения $H \psi=E \psi+O\left(h^{2}\right)$. Отсюда с использованием леммы аналогично одномерному случаю получаем утверждение теоремы.

4.3. Двумерный случай. Рассмотрим при $h \rightarrow 0$ спектральную задачу на двумерной сфере $S_{a}^{2}$ радиуса $a$ :

$$
\left(-\frac{h^{2}}{2} \Delta+\alpha \delta\left(x-x_{0}\right)\right) \psi=E \psi, \quad x \in S_{a}^{2} .
$$

Как и в трехмерном случае, квазиклассические спектральные серии выражаются в терминах классического объекта - лагранжева многообразия $\Lambda$, которое описывает классические движения, соответствующие квантовой задаче (41). Это многообразие определяется аналогично трехмерному случаю. Пусть $(x, p) \in T^{*} S_{a}^{2}$, где $T^{*} S_{a}^{2}-$ кокасательное расслоение к сфере. Рассмотрим траектории (см. рис. 3 )

$$
x=X(\omega, t), \quad p=P(\omega, t), \quad \omega \in S_{\sqrt{2 E}}^{1}, \quad t \in \mathbb{R},
$$

гамильтоновой системы

$$
\dot{x}=\frac{\partial H}{\partial p}, \quad \dot{p}=-\frac{\partial H}{\partial x}, \quad H=\frac{p^{2}}{2},
$$

с начальными условиями

$$
x(0)=x_{0}, \quad p(0)=\omega, \quad|\omega|=\sqrt{2 E} ;
$$

здесь $S_{\sqrt{2 E}}^{1}-$ окружность радиуса $\sqrt{2 E}$. Другими словами, из точки $x_{0}$ (в которой находится дельта-потенциал) по поверхности сферы $S_{a}^{2}$ с импульсом $p \in \Lambda_{0}$, где $\Lambda_{0}=\left\{x=x_{0},|p|=\sqrt{2 E}\right\}$, выпускаются траектории. Получается многообразие 


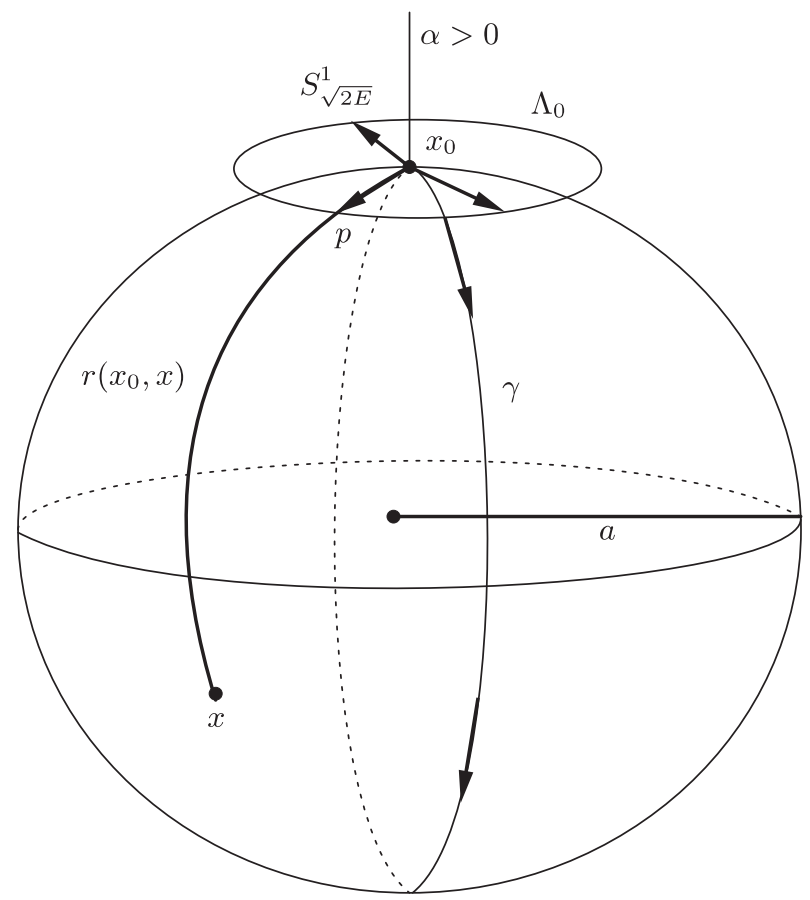

Рис. 3. Классические траектории на сфере $S_{a}^{2}$, соответствующие квантовой задаче (41).

$\Lambda=\bigcup_{t} g_{t} \Lambda_{0}$, диффеоморфное $S^{1} \times S^{1}$ (рис. 4). Это многообразие описывает нужные классические движения. Проекции траекторий на сферу $S_{a}^{2}$ суть геодезические.

Многообразие $\Lambda$ имеет два цикла $\gamma$ и $\gamma_{0}$, где $\gamma_{0}$ - цикл на начальном многообразии $\Lambda_{0}$, условие квантования на котором выполнено автоматически, так как $\left.d x\right|_{\Lambda_{0}}=0\left(x=x_{0}=\right.$ const на $\left.\Lambda_{0}\right)$. Поэтому вклад в спектр дают лишь интегралы по циклу $\gamma$. В качестве этого цикла можно выбрать замкнутую траекторию гамильтоновой системы (43) с начальными условиями (44) (рис. 3).

Проекция многообразия $\Lambda$ на $x$-пространство устроена аналогично случаю трехмерной сферы. В каждую точку сферы $S_{a}^{2}$ за исключением точки $x_{0}$ и диаметрально противоположной ей точки $x^{\prime}$ проецируются две точки многообразия $\Lambda$, а именно точки $(x, p)$ и $(x,-p)$. В точки $x_{0}$ и $x^{\prime}$ проецируется вся окружность $S_{\sqrt{2 E}}^{1}$. Условие квантования на многообразии $\Lambda$ по циклу $\gamma$ и есть искомое условие на спектр задачи (41).

Если из многообразия $\Lambda$ выкинуть окружность $S_{\sqrt{2 E}}^{1}$ над точкой $x_{0}$, то мы получим некомпактное многообразие $\Lambda^{\prime}=\Lambda \backslash S^{1}$, которое определяет собственную функцию.

ТЕОРема 3. Пусть число $E=O(1)$ удовлетворяет следующему уравнению:

$$
\operatorname{tg}\left(\frac{1}{2 h} \oint_{\gamma}(p, d x)+O(h)\right)=\frac{2}{\pi}\left(\ln \left(\frac{1}{h} \sqrt{\frac{E}{2}}+O(h)\right)+c+\frac{\pi h^{2}}{\alpha}\right)
$$




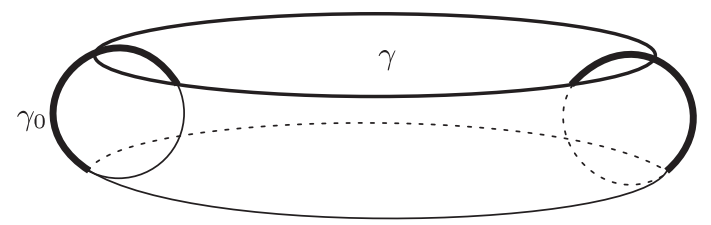

Рис. 4. Лагранжево многообразие $\Lambda$, диффеоморфное $S^{1} \times S^{1}$, соответствующее квантовой задаче (41).

которое может быть записано в следующем виде: $п р и \frac{h^{2}}{\alpha}+\frac{\ln h^{-1}}{\pi} \rightarrow \infty$

$$
\oint_{\gamma}(p, d x)=2 \pi h\left(k+\frac{1}{2}\right)
$$

где $k \in \mathbb{Z}_{+}, k \sim 1 / h$ при $h \rightarrow 0 ;$ nрu $\alpha=\frac{\pi h^{2}}{\ln h+B}, B=$ const,

$$
\operatorname{tg}\left(\frac{1}{2 h} \oint_{\gamma}(p, d x)\right)=\frac{2}{\pi}\left(\ln \sqrt{\frac{E}{2}}+c+B\right),
$$

где с - постоянная Эйлера.

Тогда существуют собственное значение $E_{0}$ оператора $H$ такое, что $\left|E-E_{0}\right|=$ $o(h)$, и функция $\psi$ такая, что $H \psi=E \psi+o(h)$. Функция $\psi$ при $r\left(x, x_{0}\right) \geqslant \varepsilon$ $u r\left(x, x^{\prime}\right) \geqslant \varepsilon($ әде $\varepsilon>0$ не зависит от $h$ ) выражается через канонический оператор Маслова на многообразии $\Lambda^{\prime}: \psi=K_{\Lambda^{\prime}}[1]$. Вне $\varepsilon$-окрестностей точек $x_{0}$ и $x^{\prime}$ собственная функиия определяется выражением

$$
\begin{aligned}
\psi= & \frac{C_{1}}{\sqrt{a \sin \frac{r\left(x, x_{0}\right)}{a}}} \exp \left(\frac{i \pi a \sqrt{2 E}}{h}+\frac{i \pi}{4}\right) \times \\
& \times \cos \left(\frac{1}{2 h} \int_{x_{0}}^{x}(p, d x)-\frac{\pi a \sqrt{2 E}}{h}-\frac{\pi}{4}\right)+O(h) ;
\end{aligned}
$$

при $x \rightarrow x_{0}$ функиия имеет логарифмическую особенность: $\psi \sim C_{2} \ln \frac{r\left(x, x_{0}\right)}{a} ;$ здесь $C_{1}, C_{2}=$ const.

ЗАмечаниЕ 5. Интегралы в теореме 3 можно вычислить: имеем, соответственно, для уравнений (45), (46)

$$
\begin{aligned}
& E=\frac{h^{2}}{2 a^{2}}\left(k+\frac{1}{2}\right)^{2} \quad \text { при } \quad \frac{h^{2}}{2 \alpha}-\frac{1}{\pi} \ln h \rightarrow \infty ; \\
& \operatorname{tg}\left(\frac{\pi a}{h} \sqrt{2 E}\right)=\frac{2}{\pi}\left(\ln \sqrt{\frac{E}{2}}+c+B\right) \quad \text { при } \quad \alpha=\frac{\pi h^{2}}{\ln h+B} .
\end{aligned}
$$

ЗАмечАние 6 . Наличие особенности у функции $\psi$ является следствием некомпактности многообразия $\Lambda^{\prime}$. 
ЗАмечАниЕ 7. Как видно из теоремы 3, результат для двумерного случая не является полным аналогом результата для трехмерного случая. При почти всех значениях $\alpha$ условия квантования совпадают со стандартными условиями Бора-Зоммерфельда. Нестандартные условия появляются при специальной нормировке дельтапотенциала: коэффициент должен равняться $\pi h^{2} /(\ln h+B)$. Отметим, что специальная нормировка похожего вида в двумерном случае возникает и при аппроксимации дельта-потенциалов гладкими функциями [10].

ДокАзАтЕльство. Точное уравнение на спектр в случае двумерной сферы имеет вид (12), где

$$
\begin{aligned}
Q\left(E ; x_{0}\right)= & -\frac{1}{2 \pi}\left\{\omega\left(\frac{1}{2}+\frac{a \sqrt{2 E}}{h} \sqrt{1+\frac{h^{2}}{8 a^{2} E}}\right)+c-\right. \\
& \left.-\frac{\pi}{2} \operatorname{tg}\left(\frac{1}{2}+\frac{a \sqrt{2 E}}{h} \sqrt{1+\frac{h^{2}}{8 a^{2} E}}\right)-\ln 2 a\right\}
\end{aligned}
$$

- Q-функция Крейна для двумерной сферы $S_{a}^{2}$ [12].

Теперь получим асимптотику выражения (47) при $h \rightarrow 0$. Учитывая, что

$$
\left.\left(\frac{1}{2}+\frac{a \sqrt{2 E}}{h} \sqrt{1+\frac{h^{2}}{8 a^{2} E}}\right)\right|_{h \rightarrow 0}=\frac{a \sqrt{2 E}}{h}+O(h),
$$

а также используя разложение при $h \rightarrow 0$ [22]

$$
\begin{aligned}
\omega\left(\frac{a \sqrt{2 E}}{h}+O(h)\right) & =\ln \left(\frac{a \sqrt{2 E}}{h}+O(h)\right)-\frac{1}{2} \frac{1}{a \sqrt{2 E} / h+O(h)}= \\
& =\ln \left(\frac{a \sqrt{2 E}}{h}+O(h)\right)+O(h),
\end{aligned}
$$

приходим к следующей асимптотике условия квантования (12) с точностью $о(1)$ при $h \rightarrow 0:$

$$
-\frac{1}{2 \pi}\left\{\ln \left(\frac{1}{h} \sqrt{\frac{E}{2}}+O(h)\right)+c-\frac{\pi}{2} \operatorname{tg}\left(\frac{\pi a \sqrt{2 E}}{h}+O(h)\right)\right\}-\frac{h^{2}}{2 \alpha}=0
$$

или, в более привычной форме,

$$
\operatorname{tg}\left(\frac{\pi a \sqrt{2 E}}{h}+O(h)\right)=\frac{2}{\pi}\left\{\ln \left(\frac{1}{h} \sqrt{\frac{E}{2}}+O(h)\right)+c+\frac{\pi h^{2}}{\alpha}\right\} .
$$

Рассматривая данное условие квантования при различных значениях правой части, получаем условия на спектр, описанные в теореме. 
Вне конечной окрестности точки $x_{0}$ согласно формуле (13) точное выражение для собственной функции, соответствующей решениям $z=E$ уравнения (12), на двумерной сфере выражается через функцию Лежандра первого рода [12]:

$$
\psi\left(x ; x_{0}, h, E\right)=\frac{1}{4 \cos \left(\pi I_{1}(h, E)\right)} P_{-1 / 2+I_{1}(h, E)}\left(-\cos \frac{r\left(x, x_{0}\right)}{a}\right),
$$

где

$$
I_{1}(h, E)=\frac{a \sqrt{2 E}}{h} \sqrt{1+\frac{h^{2}}{8 a^{2} E}},\left.\quad I_{1}(h, E)\right|_{h \rightarrow 0}=\frac{a \sqrt{2 E}}{h}+O(h) .
$$

Используя выражение $P_{\nu}(-z)=e^{\mp i \nu \pi} P_{\nu}(z)-(2 / \pi) \sin (\pi \nu) Q_{\nu}(z)$, где $Q_{\nu}(z)$ - функция Лежандра второго рода (знак плюс в $e^{\mp i \nu \pi}$ выбирается при $\operatorname{Im} z>0$, знак минус - при $\operatorname{Im} z<0$, см., например, справочник [23]), а также разложения функций Лежандра $P_{\nu}(\cos \theta), Q_{\nu}(\cos \theta)$ при $\nu \rightarrow \infty$, получаем при $r\left(x, x_{0}\right) \geqslant \varepsilon>0$ следующую асимптотику для собственной функции (49) при $h \rightarrow 0$ :

$$
\begin{aligned}
\psi\left(x ; x_{0}, h, E\right)= & \frac{C_{3}}{\cos \frac{\pi a \sqrt{2 E}}{h} \sqrt{\sin \frac{r\left(x, x_{0}\right)}{a}}} \times \\
& \times \cos \left(\frac{r\left(x, x_{0}\right) \sqrt{2 E}}{h}-\frac{\pi a \sqrt{2 E}}{h}-\frac{\pi}{4}\right)+O(h),
\end{aligned}
$$

где $C_{3}=$ const. Известно [23], что функция Лежандра первого рода $P_{\nu}(x)$ в окрестности точки $x=-1$ (при $x \rightarrow x_{0}$ аргумент функции Лежандра в выражении (49) как раз стремится к -1 ) имеет логарифмическую особенность. Таким образом, при $x \rightarrow x_{0}$ собственная функция $\psi\left(x ; x_{0}, h, E\right)$, соответствующая собственному значению, являющемуся решением уравнения (48), при $h \rightarrow 0$ имеет асимптотику $\psi \sim C_{2} \ln \frac{r\left(x, x_{0}\right)}{a}$, следовательно, в окрестности точки $x_{0}$ для нее справедливо разложение (5)-(7), откуда мы заключаем, что $\psi\left(x ; x_{0}, h, E\right) \in D(H)$.

При выполнении условий на спектр, сформулированных в теореме, функция $\psi\left(x ; x_{0}, h, E\right)$ вне конечной окрестности точки $x_{0}$ будет выражаться через канонический оператор Маслова: $\psi=K_{\Lambda^{\prime}}[1]$ на многообразии $\Lambda^{\prime}$. Проверим это. Методом канонического оператора Маслова [17] построим решение уравнения

$$
-\frac{h^{2}}{2} \Delta \psi=E \psi+O\left(h^{2}\right)
$$

в неособых картах, а именно вне конечных окрестностей точек $x_{0}$ и $x^{\prime}$ (см. рис. 5). Аналогично трехмерному случаю неособые карты на многообразии $\Lambda$ выбираются так, чтобы точки многообразия из первой и второй карт имели противоположные импульсы. Это также соответствует тому, что из точки $x_{0} \in S_{a}^{2}$ в любую точку $x \in S_{a}^{2}$ можно попасть, двигаясь по сфере, по двум траекториям. В итоге 


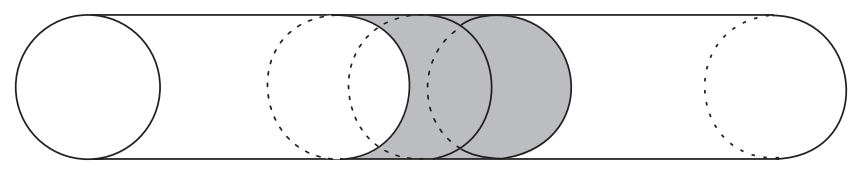

Рис. 5. Карты на лагранжевом многообразии $\Lambda^{\prime}$, диффеоморфном $\mathbb{R} \times S^{1}$. Серый цвет соответствует особой карте - окрестности точки $x^{\prime}\left(x^{\prime}\right.$ - точка, диаметрально противоположная точке $x_{0}$ ).

аналогично трехмерному случаю получаем выражение для канонического оператоpa $K_{\Lambda^{\prime}}[1]$ :

$$
K_{\Lambda^{\prime}}[1]=\frac{2}{\sqrt{J}} \exp \left(\frac{i \pi a \sqrt{2 E}}{h}+\frac{i \pi}{4}\right) \cos \left(\frac{r\left(x, x_{0}\right) \sqrt{2 E}}{h}-\frac{\pi a \sqrt{2 E}}{h}-\frac{\pi}{4}\right),
$$

где

$$
r\left(x, x_{0}\right) \sqrt{2 E}=\int_{x_{0}}^{x}(p, d x)
$$

- интеграл вдоль траектории $\gamma$,

$$
\pi a \sqrt{2 E}=\int_{x_{0}}^{x^{\prime}}(p, d x)
$$

$J=a \sin \frac{r\left(x, x_{0}\right)}{a}-$ якобиан. При $x \rightarrow x_{0}$ функция $\psi=K_{\Lambda^{\prime}}[1]$ имеет особенность вида $r^{-1 / 2}\left(x, x_{0}\right)$, следовательно, мы не можем выбирать ее в качестве собственной функции оператора $H$ в окрестности точки $x_{0}$, поскольку согласно разложению (5)-(7) в окрестности точки $x_{0}$ собственная функция из области определения оператора $H$ должна иметь логарифмическую особенность.

Конструкция канонического оператора (52) с точностью до константы совпадает с (50), таким образом, мы имеем равенство $\psi=K_{\Lambda^{\prime}}[1]$ с точностью $O(h)$. Для функции $\psi$, удовлетворяющей этому уравнению, выполняется коммутационное соотношение (22), откуда автоматически следует справедливость равенства $H \psi=$ $E_{0} \psi+O\left(h^{2}\right)$. Таким образом, с использованием леммы аналогично предыдущим случаям получаем утверждение теоремы.

Благодарности. Авторы благодарят А. А. Толченникова за полезные обсуждения. Работа поддержана РФФИ (грант № 08-01-00726) и Министерством образования и науки РФ (гранты № 2.1.1/3704, 2.1.1/4540).

\section{Список литературы}

[1] R. de L. Kronig, W. G. Penney, Proc. R. Soc. Lond. Ser. A, 130:814 (1931), 499-513.

[2] H. Bethe, R. Peierls, Proc. R. Soc. Lond. Ser. A, 148 (1935), 146-156.

[3] M. L. Goldberger, F. Seitz, Phys. Rev., 71:5 (1947), 294-310.

[4] Я. Б. Зельдович, ФTТ, 1 (1959), 1638-1645.

[5] S. Fassari, G. Inglese, Helv. Phys. Acta, 67:6 (1994), 650-659; 69:2 (1996), 130-140; 70:6 (1997), 858-865. 
[6] V.D. Krevchik, A.B. Grunin, A.K. Aringazin, M. B. Semenov, Hadronic J. Suppl., 18 (2003), 261-294.

[7] Q.-Z. Peng, X.-D. Wang, J.-Y. Zeng, Sci. China Ser. A, 34:10 (1991), 1215-1221.

[8] В. Д. Кревчик, Р. В. Зайцев, ФТТ, 43:3 (2001), 504-507.

[9] Ф. А. Березин, Л. Д. Фаддеев, ДАН СССР, 137 (1961), 1011-1014.

[10] S. Albeverio, F. Gesztesy, R. Høegh-Krohn, H. Holden, Solvable Models in Quantum Mechanics, AMS, Providence, RI, 2005.

[11] S. Albeverio, P. Kurasov, Singular Perturbations of Differential Operators, London Math. Soc. Lecture Note Ser., 271, Cambridge Univ. Press, Cambridge, 2000.

[12] J. Brüning, V. Geyler, J. Math. Phys., 44:2 (2003), 371-405, arXiv: math-ph/0205030.

[13] J. Brüning, V. Geyler, K. Pankrashkin, Rev. Math. Phys., 20:1 (2008), 1-70, arXiv: math-ph/0611088.

[14] Й. Брюнинг, В. А. Гейлер, ТМФ, 119:3 (1999), 368-380.

[15] И. С. Лобанов, Спектральные свойства гамильтонианов явнорешаемых моделей мезоскопических структур: декорированные графы и квантовые точки, Дис. ... канд. физ.-матем. наук, Мордовский гос. ун-т им. Н. П. Огарева, Саранск, 2005.

[16] В. П. Маслов, Асимптотические методы и теория возмущений, Наука, М., 1988.

[17] В.П. Маслов, М.В. Федорюк, Квазиклассическое приближение для уравнений квантовой механики, Наука, М., 1976.

[18] В. Р. Коган, Изв. вузов. Сер. Радиофизика, 12:11 (1969), 1675-1680.

[19] В. В. Кучеренко, ТМФ, 1:3 (1969), 384-406.

[20] В. А. Гейлер, В. А. Маргулис, И. И. Чучаев, Сиб. матем. журн., 36:4 (1995), 828-841.

[21] Л. Д. Ландау, Е. М. Лифшиц, Теоретическая физика, т. III, Квантовая механика. Нерелятивистская теория, Наука, М., 2001.

[22] Е. Янке, Ф. Эмде, Ф. Леш, Специальные функиии. Формуль, графики, таблиць, Наука, М., 1964.

[23] Г. Бейтмен, А. Эрдейи, Высшие трансцендентные функиии, т. I, Гипергеометрическая функция. Функции Лежандра, Наука, М., 1973.

Поступила в редакцию 13.02.2010 